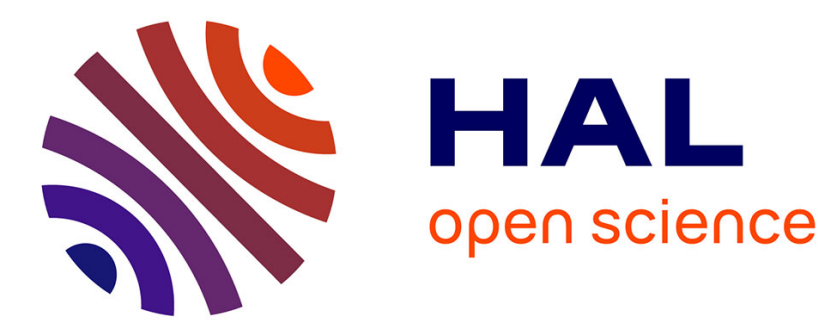

\title{
Existence of equilibrium in OLG economies with increasing returns
}

\author{
Jean-Marc Bonnisseau, Lalaina Rakotonindrainy
}

\section{To cite this version:}

Jean-Marc Bonnisseau, Lalaina Rakotonindrainy. Existence of equilibrium in OLG economies with increasing returns. 2011. halshs-00654013

\section{HAL Id: halshs-00654013 \\ https://shs.hal.science/halshs-00654013}

Submitted on 20 Dec 2011

HAL is a multi-disciplinary open access archive for the deposit and dissemination of scientific research documents, whether they are published or not. The documents may come from teaching and research institutions in France or abroad, or from public or private research centers.
L'archive ouverte pluridisciplinaire HAL, est destinée au dépôt et à la diffusion de documents scientifiques de niveau recherche, publiés ou non, émanant des établissements d'enseignement et de recherche français ou étrangers, des laboratoires publics ou privés. 


\section{Documents de Travail du Centre d'Economie de la Sorbonne}

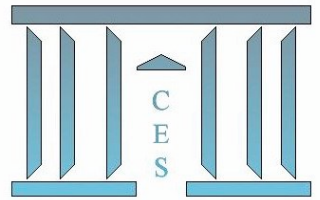

Existence of equilibrium in OLG economies

with increasing returns

Jean-Marc BONNISSEAU, Lalaina RAKOTONINDRAINY

2011.70

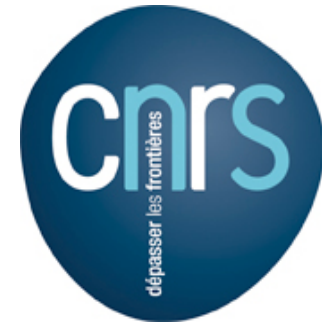




\title{
Existence of equilibrium in OLG economies with increasing returns
}

\author{
Jean-Marc Bonnisseau and Lalaina Rakotonindrainy*
}

November 2011

\begin{abstract}
We consider a standard overlapping generation economy with a simple demographic structure with a new cohort of agents at each period with an economic activity extended over two successive periods and finitely many firms active forever. The production possibilities are described by a sequence of production mapping and the main innovation comes from the fact that we allow for increasing returns to scale of more general type of non-convexities. To describe the behavior of the firms, we consider loss-free pricing rules, which covers the case of the average pricing rule, the competitive behavior when the firms have convex production sets, and the competitive behavior with quantity constraints à la Dehez-Drèze. We prove the existence of an equilibrium under assumptions, which are at the same level of generality than the ones for the existence in an exchange economy.

JEL classification: C62, D50, D62.
\end{abstract}

Keywords: Overlapping generation model, increasing returns to scale, loss-free pricing rules, equilibrium, existence

\section{Introduction}

Overlapping generations models are studied both in microeconomics and in macroeconomics to analyze intertemporal phenomenon. These models involve infinitely many dates, goods and consumers. This double infinity is

*Paris School of Economics, Université Paris 1 Panthéon-Sorbonne, Centre d'Economie de la Sorbonne-CNRS, 106-112 Boulevard de l'Hôpital, 75647 Paris Cedex 13, France. E-mail: Jean-Marc.Bonnisseau@univ-paris1.fr, Lalaina. Rakotonindrainy@malix.univ-paris1.fr 
source of many unobservable phenomenons in Arrow-Debreu economies even if the space of goods is of infinite dimension.

Regarding the production side, if we consider endogenous growth models, externalities might be introduced for example via the level of human capital, which are source of increasing returns at the aggregate level. But these returns are not taken into account by the agents, who have a myopic behavior in the sense that they do not take into account the influence of their investment in human capital on the productivity of the firms.

We thus plan to study a standard overlapping generation model with production allowing increasing returns to scale and a behavior of the producers, which goes beyond the competitive one.

The basic model is the one introduced [1, 2, 3], see also [14] for a very intuitive approach. The production knowledge of a producer is described by generalized production correspondences, which define the possible outputs at one date given the vector of inputs consumed at the previous date. This sequential approach of the production allows to consider innovation along the time and heterogeneity of producers.

The equilibrium concept is the standard one but for the behavior of the producers since we do not assume that the production sets are convex. Hence the standard competitive behavior is meaningless.

In models allowing for non-convex technologies, the firms follow general pricing rules to describe a large range of possible behaviors including the profit maximizing behavior at given prices. The literature considers pricing rule which associates a set of admissible prices to a weakly efficient production. For a comprehensive introduction see $[4,7,10,15]$. Since the production is defined in a recursive way, we propose to define also the pricing rule recursively, so that the prices for two successive dates depends on the production possibilities for these two dates and not for the other ones.

We consider loss-free pricing rules, meaning that the firms are restricted to get a non negative profit over two successive periods. This covers the case of the average pricing rule, the competitive behavior when the firms have convex production sets, and the competitive behavior with quantity constraints à la Dehez-Drèze, [8,9].

Contrary to the case of a constant return technology, it is crucial to determine how profits (or losses) of producers are distributed among consumers. Indeed, the optimality of the equilibrium allocation depends on the repartition scheme. In this first paper, we only consider private ownership economies and we assume that the shares are given exogenously. It would be meaningful to introduce a stock market at each date allowing the old generation to 
sell the shares to the young generation, but this is beyond the scope of this paper.

We provide an existence result under sufficient conditions at the same level of generality than those for an exchange economy. On the production side, we need to assume the free-disposal condition as for the static models. On the pricing rule, we just need a continuity condition.

\section{Description of the model}

We consider an economy with infinitely many dates $(t=1,2 \cdots)$. For all $t \in \mathbb{N}^{*}$, there exists a finite set $\mathcal{L}_{t}$ of commodities available in the world. We denote $\# \mathcal{L}_{t}=L_{t}$.

\section{Consumers}

At each period $t \in \mathbb{N}$ (including at period 0 ), a finite and non-empty set of consumers $\mathcal{I}_{t}$, called generation $t$, born. We denote $\# \mathcal{I}_{t}=I_{t}$ and $\mathcal{I}=\cup_{t \in \mathbb{N}} \mathcal{I}_{t}$. Each individual lives two periods (an agent born at period $t$ lives at $t$ and $t+1$ and is assumed to have no economic activity before $t$ and after $t+1$ ).

The consumption set of each individual $i \in \mathcal{I}_{t}, t \geq 1$ is the subset $X^{i}=$ $\mathbb{R}_{+}^{L_{t}} \times \mathbb{R}_{+}^{L_{t+1}}$. Thus consumption of each consumer of generation $t$ is limited to his lifetime $t$ and $t+1$. The consumption set of consumers of generation 0 is $\mathbb{R}_{+}^{L_{1}}$.

Consumers preferences are represented by a utility function $u^{i}: X^{i} \rightarrow \mathbb{R}$. This means that preferences are complete and transitive.

The vector $e^{i} \in \mathbb{R}_{++}^{L_{t}} \times \mathbb{R}_{++}^{L_{t+1}}$ represents the initial endowment of the agent $i$ of the generation $t$, which is null outside his lifetime.

\section{Producers}

We assume the set of producers $\mathcal{J}$ to be finite. Each firm is supposed to be possibly active for all dates. We denote $\# \mathcal{J}=J$.

The production possibilities are represented by production mappings associating to a given vector of inputs at date $t$, a set of possible outputs produced at the next period. This supposes that the production process takes time, the consumption of an input at date $t$ has no influence on the output at this date. For each firm $j,\left(F_{t}^{j}\right)_{t=1}^{\infty}$ is a sequence of mappings from $-\mathbb{R}_{+}^{L_{t}}$ to $\mathbb{R}^{L_{t+1}}$. For a given inputs vector $z_{t}^{j}, F_{t}^{j}\left(z_{t}^{j}\right)$ is the set of possible vector of outputs the firm can produce.

Let us associate to each firm $j$ at each period $t$ an elementary production 
set $Z_{t}^{j}$ defined by:

$$
Z_{t}^{j}=\left\{\left(z_{t}^{j}, \zeta_{t+1}^{j}\right) \in-\mathbb{R}_{+}^{L_{t}} \times \mathbb{R}^{L_{t+1}} \mid \zeta_{t+1}^{j} \in F_{t}^{j}\left(z_{t}^{j}\right)\right\}
$$

Notice that $Z_{j}^{t}$ is the graph of the mapping $F_{t}^{j}$. We define the global intertemporal production set of firm $j$ by:

$$
Y^{j}=\left\{\left(y_{t}^{j}\right)_{t=1}^{\infty} \in \prod_{t=1}^{\infty} \mathbb{R}^{L_{t}} \mid \forall t, \exists\left(z_{t}^{j}, \zeta_{t+1}^{j}\right) \in Z_{t}^{j}: y_{t}^{j}=z_{t}^{j}+\zeta_{t}^{j} \text { with } \zeta_{1}^{j}=0\right\}
$$

\section{Feasibility condition}

An allocation $\left(\left(x^{i}\right)_{i \in \mathcal{I}},\left(y^{j}\right)_{j \in \mathcal{J}}\right) \in \prod_{t=0}^{\infty} \prod_{i \in \mathcal{I}_{t}} X^{i} \times \prod_{j \in \mathcal{J}} Y^{j}$ is feasible if for all $t \in \mathbb{N}^{*}$ :

$$
\sum_{i \in \mathcal{I}_{t-1} \cup \mathcal{I}_{t}} x_{t}^{i}=\sum_{i \in \mathcal{I}_{t-1} \cup \mathcal{I}_{t}} e_{t}^{i}+\sum_{j \in \mathcal{J}} y_{t}^{j}
$$

We denote by $\mathcal{A}(\mathcal{E})$ the set of feasible allocations.

\section{Pricing Rule}

The price vector $p$ is an element of $\prod_{t=1}^{\infty} \mathbb{R}_{+}^{L_{t}}$, and $p_{t h}$ is the market price of the commodity $h$ at date $t$.

Since the model we consider allows increasing returns, the producers behavior cannot only be characterized by a competitive and profit maximization behavior. So we describe the behavior of the producers by general pricing rules. See Cornet [7], Dierker, Guesnerie and Neuefeind [10] and Villar [15] for a survey on the representation of economic behavior of producers by pricing rules.

Since the production possibilities are defined in a recursive way, we define the pricing rule in a similar way. For a producer $j$ at a period $t$, the pricing rule $\varphi_{t}^{j}$ is a set-valued mapping defined on the set of weakly efficient productions of $Z_{t}^{j}$ with values in $\mathbb{R}_{+}^{L_{t}} \times \mathbb{R}_{+}^{L_{t+1}}$. So, taken a weakly efficient production $y^{j} \in Y^{j}$ and a price $p$, the pair $\left(y^{j}, p\right)$ is compatible with the behavior of the $j$ th producer if for all $t,\left(p_{t}, p_{t+1}\right) \in \varphi_{t}^{j}\left(z_{t}^{j}, \zeta_{t+1}^{j}\right)$ where $\left(z_{t}^{j}, \zeta_{t+1}^{j}\right) \in Z_{t}^{j}$ and $y_{t}^{j}=z_{t}^{j}+\zeta_{t}^{j}$.

\section{Budget Constraint}

We assume that we are in a private ownership economy. Each agent $i \in \mathcal{I}_{t}$ beholds a share $\theta^{i j} \geq 0$ of the firm $j$ such that for all $j, \sum_{i \in \mathcal{I}_{t}} \theta^{i j}=1$. 
The budget constraint, for each agent $i \in \mathcal{I}_{t}, t \in \mathbb{N}^{*}$ is given by:

$$
p_{t} \cdot x_{t}^{i}+p_{t+1} \cdot x_{t+1}^{i} \leq p_{t} \cdot e_{t}^{i}+p_{t+1} \cdot e_{t+1}^{i}+\sum_{j \in \mathcal{J}} \theta^{i j}\left(p_{t} \cdot z_{t}^{j}+p_{t+1} \cdot \zeta_{t+1}^{j}\right)
$$

and for $i \in \mathcal{I}_{0}, p_{1} \cdot x_{1}^{i} \leq p_{1} \cdot e_{1}^{i}$

\section{Equilibrium}

We are now able to state the definition of an equilibrium in this overlapping generation economy with production.

Definition 1 An equilibrium in the OLG economy $\mathcal{E}$ is an element $\left(p^{*},\left(x^{i *}\right),\left(y^{j *}\right)\right) \in \prod_{t=1}^{\infty} \mathbb{R}_{+}^{L_{t}} \times \prod_{i \in \mathcal{I}} X^{i} \times \prod_{j \in \mathcal{J}} Y^{j}$ such that:

a) for all $t \in \mathbb{N}^{*}$, for all $i \in \mathcal{I}_{t}, x^{i *}$ is a maximal element of $u^{i}$ in the budget set:

$$
\left\{x^{i} \in X^{i} \mid p_{t}^{*} \cdot x_{t}^{i}+p_{t+1}^{*} \cdot x_{t+1}^{i} \leq w^{i *}\right\}
$$

with $w^{i *}=p_{t}^{*} \cdot e_{t}^{i}+p_{t+1}^{*} \cdot e_{t+1}^{i}+\sum_{j \in \mathcal{J}} \theta^{i j}\left(p_{t}^{*} \cdot z_{t}^{j}+p_{t+1}^{*} \cdot \zeta_{t+1}^{j}\right)$ and, for all $i \in \mathcal{I}_{0}$, $x^{i *}$ is a maximal element of $u^{i}$ in the budget set $\left\{x^{i} \in X^{i} \mid p_{1}^{*} \cdot x_{1}^{i} \leq p_{1}^{*} \cdot e_{1}^{i}\right\} ;$

b) for all $j \in \mathcal{J}$, for all $t,\left(p_{t}^{*}, p_{t+1}^{*}\right) \in \varphi_{t}^{j}\left(z_{t}^{j *}, \zeta_{t+1}^{j *}\right)$, where $\left(z_{t}^{j *}, \zeta_{t+1}^{j *}\right) \in Z_{t}^{j}$ and $y_{t}^{j *}=z_{t}^{j *}+\zeta_{t}^{j *}$

c) for all $t \in \mathbb{N}^{*}, \sum_{i \in \mathcal{I}_{t-1} \cup \mathcal{I}_{t}} x_{t}^{i *}=\sum_{i \in \mathcal{I}_{t-1} \cup \mathcal{I}_{t}} e_{t}^{i}+\sum_{j \in \mathcal{J}} y_{t}^{j *}$.

\section{Existence of equilibrium}

We consider standard assumptions on the consumption side.

\section{Assumption C.}

a) For all $t \in \mathbb{N}^{*}$, for all individuals $i \in \mathcal{I}_{t}, X^{i}=\mathbb{R}_{+}^{L_{t}} \times \mathbb{R}_{+}^{L_{t}+1}$ and for all $i \in \mathcal{I}_{0}, X^{i}=\mathbb{R}_{+}^{L_{1}}$.

b) For all individuals in $\mathcal{I}, u^{i}$ is continuous, quasi-concave and locally nonsatiated;

c) For all $t \in \mathbb{N}^{*}$, there exists $i_{0}(t) \in \mathcal{I}_{t}$ such that for all $x_{t} \in \mathbb{R}_{+}^{L_{t}}, u^{i_{0}(t)}\left(x_{t}, \cdot\right)$ is locally non-satiated and $i_{1}(t) \in \mathcal{I}_{t}$ such that for all $x_{t+1} \in \mathbb{R}_{+}^{L_{t+1}}$, $u^{i_{1}(t)}\left(\cdot, x_{t+1}\right)$ is locally non-satiated. 
Assumption E. For all $t \in \mathbb{N}^{*}$, for all $i \in \mathcal{I}_{t}, e^{i} \in \mathbb{R}_{++}^{L_{t}} \times \mathbb{R}_{++}^{L_{t}+1}$ and for all $i \in \mathcal{I}_{0}, e^{i} \in \mathbb{R}_{++}^{L_{1}}$.

We posit the following assumption on the production function.

\section{Assumption F.}

a) For all $(j, t) \in \mathcal{J} \times \mathbb{N}^{*}, F_{t}^{j}$ has a closed graph;

b) for all $z_{t}^{j} \in-\mathbb{R}_{+}^{L_{t}}, 0 \in F_{t}^{j}\left(z_{t}^{j}\right)$;

c) for all $z_{t}^{j} \in-\mathbb{R}_{+}^{L_{t}}, F_{t}^{j}\left(z_{t}^{j}\right) \cap \mathbb{R}_{+}^{L_{t+1}}$ is bounded;

d) for all $z_{t}^{j}, z_{t}^{j \prime} \in-\mathbb{R}_{+}^{L_{t}}$, if $z_{j}^{t} \leq z_{t}^{j \prime}$ then $F_{t}^{j}\left(z_{t}^{j \prime}\right) \subset F_{t}^{j}\left(z_{j}^{t}\right)$;

e) $F_{t}^{j}\left(z_{t}^{j}\right)=\left(F_{t}^{j}\left(z_{t}^{j}\right) \cap \mathbb{R}_{+}^{L_{t+1}}\right)-\mathbb{R}_{+}^{L_{t+1}}$.

Assumption $\mathrm{F}$ implies that $Y^{j}$ is closed for the product topology and satisfies the free-disposal assumption and the inactivity property. We do not assume the output vectors to be nonnegative, but we will see that only the nonnegative output vectors are relevant at equilibrium.

Assumption PR. For all $(j, t) \in \mathcal{J} \times \mathbb{N}^{*}$,

a) $\varphi_{t}^{j}$ has a closed graph and for all $\left(z_{t}^{j}, \zeta_{t+1}^{j}\right) \in \partial Z_{t}^{j}, \varphi_{t}^{j}\left(z_{t}^{j}, \zeta_{t+1}^{j}\right)$ is a closed convex cone in $\mathbb{R}_{+}^{L_{t}} \times \mathbb{R}_{+}^{L_{t+1}}$ different from $\{(0,0)\}$;

b) for all $\left(z_{t}^{j}, \zeta_{t+1}^{j}\right) \in \partial Z_{t}^{j}$, for all $\left(p_{t}, p_{t+1}\right) \in \varphi_{t}^{j}\left(z_{t}^{j}, \zeta_{t+1}^{j}\right)$, if $\zeta_{t+1, k}<0$ then $p_{t+1, k}=0$.

Assumption LF. (Loss-free assumption) For all $(j, t) \in \mathcal{J} \times \mathbb{N}$, for all $\left(z_{t}^{j}, \zeta_{t+1}^{j}\right) \in Z_{t}^{j}$, for all $\left(p_{t}, p_{t+1}\right) \in \varphi_{t}^{j}\left(z_{t}^{j}, \zeta_{t+1}^{j}\right)$,

$$
p_{t} \cdot z_{t}^{j}+p_{t+1} \cdot \zeta_{t+1}^{j} \geq 0
$$

The main result of this paper is the following:

Theorem 1 Under Assumptions $C, E, F, P R$ and $L F$, the $O L G$ economy $\mathcal{E}$ has an equilibrium.

Remark 1 This result encompasses the known existence results for exchange economies. Indeed, it suffices to consider that there is only one producer with a constant production correspondence $F_{t}$ defined by $F_{t}\left(z_{t}\right)=-\mathbb{R}_{+}^{L_{t+1}}$ and the pricing rule corresponding to the competitive behavior, that is, $\varphi_{t}\left(z_{t}, \zeta_{t+1}\right)=$ $\left\{\left(p_{t}, p_{t+1}\right) \in \mathbb{R}_{+}^{L_{t}} \times \mathbb{R}_{+}^{L_{t+1}} \mid p_{t} \cdot z_{t}+p_{t+1} \cdot \zeta_{t+1}=0\right\}$. 
Remark 2 If we further assume that $F_{t}^{j}$ has a convex graph for all $(j, t)$ and that the pricing rule $\varphi_{t}^{j}$ describes the competitive behavior, that is, $\varphi_{t}^{j}\left(z_{t}^{j}, \zeta_{t+1}^{j}\right)=\left\{\left(p_{t}, p_{t+1}\right) \in \mathbb{R}_{+}^{L_{t}} \times \mathbb{R}_{+}^{L_{t+1}} \mid p_{t} \cdot z_{t}^{j}+p_{t+1} \cdot \zeta_{t+1}^{j} \geq p_{t} \cdot z_{t}^{j \prime}+\right.$ $\left.p_{t+1} \cdot \zeta_{t+1}^{j \prime}, \forall\left(z_{t}^{j \prime}, \zeta_{t+1}^{j \prime}\right) \in Z_{t}^{j}\right\}$, then Assumptions PR and LF are satisfied and Theorem 1 gives the existence of a competitive equilibrium in the OLG economy.

Remark 3 Note that Assumption PR (b) implies that for all $t \in \mathbb{N}^{*}$, for all $k \in \mathcal{L}_{t+1}$, then $\zeta_{t+1, k}^{j *}=0$ if commodity $k$ is desirable by at least one consumer of generation $t$ or $t+1$. So, even if we do not a priori exclude negative quantities of output when we define the production mappings, at equilibrium, the production of an output is always non negative for desirable commodities.

\section{Equilibrium in truncated economies}

We will proceed as in exchange economies (see Balasko et al. [1]) to establish the existence of equilibrium in $\mathcal{E}$ : first we show the existence of pseudoequilibrium in the truncated economies with a finite horizon

$$
\mathcal{E}_{\tau}=\left(\left(u^{\tau i}, X^{\tau i}, e^{\tau i}, \theta^{i}\right)_{i \in \mathcal{I}_{0}^{\tau-1}},\left(Y^{t j}, \tilde{\varphi}^{t j}\right)_{\substack{t=1, \ldots, \tau-1 \\ j \in \mathcal{J}}}\right)
$$

then we prove that prices and allocations remains in a compact space of a suitable linear space and we finally show that a cluster point is an equilibrium of the OLG economy.

\section{Notations.}

$\mathcal{I}_{0}^{\tau-1}=\cup_{t=0}^{\tau-1} \mathcal{I}_{t}$ is the set of all the individuals born up to period $\tau-1$.

For each $t$, we choose an arbitrary closed convex cone $C_{t}$ included in $\mathbb{R}_{++}^{L_{t}} \cup\{0\}$ containing $\mathbf{1}^{t}=(1, \ldots, 1)$ in its interior. We denote by $C_{t}^{+}$the positive polar cone of $C_{t}{ }^{1}$. For each $i \in \mathcal{I}_{0}$,

$X^{\tau i}=\left\{x \in \prod_{t=1}^{\tau} \mathbb{R}_{+}^{L_{t}} \mid x_{t^{\prime}}=0, \forall t^{\prime}>1\right\}$

$u^{\tau i}(x)=u^{i}\left(x_{1}\right)$

$e^{\tau i}=\left(e_{t^{\prime}}^{\tau i}\right)_{t^{\prime}=1}^{\tau}$ such that $e_{1}^{\tau i}=e_{1}^{i}$, and $e_{t^{\prime}}^{\tau i}=0$ if $t^{\prime}>1$.

For each $t=1, \ldots, \tau-1$, for each $i \in \mathcal{I}_{t}$,

$X^{\tau i}=\left\{x \in \prod_{t=1}^{\tau} \mathbb{R}_{+}^{L_{t}} \mid x_{t^{\prime}}=0, \forall t^{\prime} \neq t, t+1\right\}$

$u^{\tau i}(x)=u^{i}\left(x_{t}, x_{t+1}\right)$

${ }^{1} C_{t}^{+}=\left\{v \in \mathbb{R}^{L_{t}} \mid v \cdot u \geq 0, \forall u \in C_{t}\right\}$ 
$e^{\tau i}=\left(e_{t^{\prime}}^{\tau i}\right)_{t^{\prime}=1}^{\tau}$ such that $e_{t}^{\tau i}=e_{t}^{i}, e_{t+1}^{\tau i}=e_{t+1}^{i}$ and $e_{t^{\prime}}^{\tau i}=0$ if $t^{\prime} \neq t, t+1$.

We define the extended production set $Y^{t j}$ for $t=1, \ldots, \tau-1$ as follows:

$$
Y^{t j}=\left\{\left(y_{t^{\prime}}^{t j}\right)_{t^{\prime}=1}^{\tau} \in \prod_{t^{\prime}=1}^{\tau} \mathbb{R}^{L_{t^{\prime}}} \mid\left(y_{t}^{t j}, y_{t+1}^{t j}\right) \in Z_{t}^{j}, \forall t^{\prime} \neq t, t+1, y_{t^{\prime}}^{t j} \in-C_{t^{\prime}}\right\}
$$

This extension is necessary since the existence result for economies with non-convex production sets require that the production sets satisfies the freedisposal assumption or at least a weak form of it, namely, with our notations the fact that $Y^{t j}-\prod_{t^{\prime}=1}^{\tau} C_{t^{\prime}}=Y^{t j}$. We also extend the pricing rules as follows: for all $y^{t j} \in \partial Y_{t j}$,

$$
\tilde{\varphi}^{t j}\left(y^{t j}\right)=\left\{p \in \prod_{t^{\prime}=1}^{\tau} C_{t}^{+} \mid\left(p_{t}, p_{t+1}\right) \in \varphi_{t}^{j}\left(y_{t}^{t j}, y_{t+1}^{t j}\right), p_{t^{\prime}} \cdot y_{t^{\prime}}^{t j}=0, \forall t^{\prime} \neq t, t+1\right\}
$$

We remark that if $p \in \tilde{\varphi}^{t j}\left(y^{t j}\right)$ and $p_{t^{\prime}} \in \mathbb{R}_{+}^{L_{t^{\prime}}} \backslash\{0\}$ for some $t^{\prime} \neq t, t+1$, then $y_{t^{\prime}}^{t j}=0$.

Definition 2 A pseudo-equilibrium in the truncated economy $\mathcal{E}_{\tau}$ is an element $\left(p^{*},\left(x^{i *}\right),\left(y^{t j *}\right)\right) \in \prod_{t=1}^{\tau} C_{t}^{+} \times \prod_{i \in \mathcal{I}_{0}^{\tau-1}} X^{\tau i} \times \prod_{j \in \mathcal{J}} \prod_{t=1}^{\tau-1} Y^{t j}$ such that:

a) for all $t=1,2 \ldots \tau-1$, for all $i \in \mathcal{I}_{t}, x^{i *}$ is a maximal element of $u^{\tau i}$ in the budget set

$$
\left\{x^{i} \in X^{\tau i} \mid p^{*} \cdot x_{i} \leq p^{*} \cdot e^{\tau i}+\sum_{j \in \mathcal{J}} \theta_{t}^{i j} p^{*} \cdot y^{t j *}\right\}
$$

for all $i \in \mathcal{I}_{0}, x^{i *}$ is a maximal element of $u^{\tau i}$ in the budget set $\left\{x^{i} \in X^{\tau i}\right.$ $\left.p^{*} \cdot x^{i} \leq p^{*} \cdot e^{\tau i}\right\}$;

b) for all $j \in \mathcal{J}$, for all $t=1, \ldots, \tau-1, p^{*} \in \tilde{\varphi}^{t j}\left(y^{t j *}\right)$;

c) For all $t=1, \ldots, \tau-1, \sum_{i \in \mathcal{I}_{0}^{\tau-1}} x_{t}^{i *}=\sum_{i \in \mathcal{I}_{0}^{\tau-1}} e_{t}^{\tau i}+\sum_{j \in \mathcal{J}} \sum_{t^{\prime}=1}^{\tau-1} y_{t}^{t^{\prime} j *}$ and $\sum_{i \in \mathcal{I}_{0}^{\tau-1}} x_{\tau}^{i *} \leq \sum_{i \in \mathcal{I}_{0}^{\tau-1}} e_{\tau}^{\tau i}+\sum_{i \in \mathcal{I}_{\tau}} e_{\tau}^{i}+\sum_{j \in \mathcal{J}} \sum_{t^{\prime}=1}^{\tau-1} y_{\tau}^{t^{\prime} j *}$

Remark 4 The difference between a pseudo-equilibrium and an equilibrium is that we do not require the market clearing condition at the last period $\tau$ and we artificially increase the initial endowments by adding those of the consumers of the generation $\tau$. This particular feature is useful to show below that if $\tau^{\prime}>\tau$, then the restriction of a pseudo-equilibrium of $\mathcal{E}_{\tau^{\prime}}$ to the $\tau-1$ first generations is a pseudo-equilibrium of $\mathcal{E}_{\tau}$.

Remark 5 Since Condition (c) of the above definition is weaker on the last period $\tau$ than the standard market clearing condition, an equilibrium of $\mathcal{E}_{\tau}$ is clearly a pseudo-equilibrium. 
Remark 6 In the definition of a pseudo-equilibrium, the price $p^{*}$ is supposed to be in $\prod_{t=1}^{\tau} C_{t}^{+}$. Actually, we remark that it belongs to the smaller set $\prod_{t=1}^{\tau} \mathbb{R}_{+}^{L_{t}}$. This is a consequence of Condition (b) and the fact that $\varphi_{t}^{j}$ takes its values in $\mathbb{R}_{+}^{L_{t}} \times \mathbb{R}_{+}^{L_{t+1}}$. Consequently, we deduce from the definition of $\tilde{\varphi}^{t j}$ that $y_{t^{\prime}}^{t j *}=0$ for all $t^{\prime} \neq t, t+1$.

Remark 7 From the definition of the truncated economy and the definition of a pseudo-equilibrium, we remark that if $\bar{\tau}>\tau$ and $\left(p^{*},\left(x^{i *}\right),\left(y^{t j *}\right)\right)$ is a pseudo-equilibrium in the economy $\mathcal{E}_{\bar{\tau}}$, then the price and the allocations restricted to the $\tau$ first periods $\left(q^{*},\left(\chi^{i *}\right)_{i \in \mathcal{I}_{0}^{\tau-1}},\left(\xi^{t j *}\right)_{\substack{t=1, \ldots, \tau-1 \\ j \in \mathcal{J}}}\right)$ defined by

$q^{*}=\left(p_{t}^{*}\right)_{t=1}^{\tau}$,

for all $i \in \mathcal{I}_{0}^{\tau-1}, \chi^{i *}=\left(x_{t}^{i *}\right)_{t=1}^{\tau}$,

for all $j \in \mathcal{J}$, for all $t=1, \ldots, \tau-1, \xi^{t j *}=\left(z_{t^{\prime}}^{t j *}\right)_{t^{\prime}=1}^{\tau}$,

is a pseudo-equilibrium in the economy $\mathcal{E}_{\tau}$.

Indeed, from the definition of a quasi-equilibrium, we just have to look at Condition (c) for the period $\tau$. Since $\left(p^{*},\left(x^{i *}\right),\left(y^{t j *}\right)\right)$ is a pseudo-equilibrium in the economy $\mathcal{E}_{\bar{\tau}}$ and $\bar{\tau}>\tau$, one has:

$$
\sum_{i \in \mathcal{I}_{0}^{\bar{\tau}-1}} x_{\tau}^{i *}=\sum_{i \in \mathcal{I}_{0}^{\bar{\tau}-1}} e_{\tau}^{\bar{\tau} i}+\sum_{j \in \mathcal{J}} \sum_{t^{\prime}=1}^{\bar{\tau}-1} y_{\tau}^{t^{\prime} j *}
$$

From the definition of $X^{\bar{\tau} i}$, for all $i \in \cup_{t=\tau+1}^{\bar{\tau}-1} \mathcal{I}_{t}, x_{\tau}^{i *}=0$. From the definition of $e^{\bar{\tau} i}$, for all $i \in \cup_{t=\tau+1}^{\bar{\tau}-1} \mathcal{I}_{t}, e_{\tau}^{\bar{\tau} i}=0$. From the previous remark, for all $t^{\prime}=\tau+1, \ldots, \bar{\tau}-1$, for all $j, y_{\tau}^{t^{\prime} j *}=0$. Furthermore, for all $j, y_{\tau}^{\tau j *} \leq 0$ and for all $i \in \mathcal{I}_{\tau}, x_{\tau}^{i *} \geq 0$. So, one deduces that

$$
\sum_{i \in \mathcal{I}_{0}^{\bar{\tau}-1}} x_{\tau}^{i *}=\sum_{i \in \mathcal{I}_{0}^{\tau-1}} x_{\tau}^{i *}+\sum_{i \in \mathcal{I}_{\tau}} x_{\tau}^{i *}=\sum_{i \in \mathcal{I}_{0}^{\tau-1}} e_{\tau}^{\bar{\tau} i}+\sum_{i \in \mathcal{I}_{\tau}} e_{\tau}^{\bar{\tau} i}+\sum_{j \in \mathcal{J}} \sum_{t^{\prime}=1}^{\tau-1} y_{\tau}^{t^{\prime} j *}+\sum_{j \in \mathcal{J}} y_{\tau}^{\tau j *}
$$

which implies that

$$
\sum_{i \in \mathcal{I}_{0}^{\tau-1}} x_{\tau}^{i *} \leq \sum_{i \in \mathcal{I}_{0}^{\tau-1}} e_{\tau}^{\bar{\tau} i}+\sum_{i \in \mathcal{I}_{\tau}} e_{\tau}^{\bar{\tau} i}+\sum_{j \in \mathcal{J}} \sum_{t^{\prime}=1}^{\tau-1} y_{\tau}^{t^{\prime} j *}
$$

So we get Condition (c) for the period $\tau$ since $x_{\tau}^{i *}=\chi_{\tau}^{i *}$ and $e_{\tau}^{\bar{\tau} i}=e_{\tau}^{\tau i}$ for all $i \in \mathcal{I}_{0}^{\tau-1}$ and $e_{\tau}^{\bar{\tau} i}=e_{\tau}^{i}$ for all $i \in \mathcal{I}_{\tau}$.

We are going to deduce the existence of pseudo-equilibrium from a quasiequilibrium. One has: 
Definition 3 A quasi-equilibrium in the truncated economy $\mathcal{E}_{\tau}$ is an element $\left(p^{*},\left(x^{i *}\right),\left(y^{t j *}\right)\right) \in \prod_{t=1}^{\tau} C_{t}^{+} \times \prod_{i \in \mathcal{I}_{0}^{\tau-1}} X^{\tau i} \times \prod_{j \in \mathcal{J}} \prod_{t=1}^{\tau-1} Y^{t j}$ satisfying:

a') for all $t=1,2 \ldots \tau-1, x^{i *}$ is an element of the budget set:

$$
\left\{x^{i} \in X^{\tau i} \mid p^{*} \cdot x^{i} \leq p^{*} \cdot e^{\tau i}+\sum_{j \in \mathcal{J}} \theta_{t}^{i j} p^{*} \cdot y^{t j *}\right\}
$$

and for all $x^{i} \in X^{\tau i}$ such that: $p^{*} \cdot x^{i}<p^{*} \cdot e^{\tau i}+\sum_{j \in \mathcal{J}} \theta_{t}^{i j} p^{*} \cdot y^{t j}, u^{\tau i}\left(x^{i}\right) \leq$ $u^{\tau i}\left(x^{i *}\right)$

for all $i \in \mathcal{I}_{0}, x^{i *} \in\left\{x^{i} \in X^{i} \mid p^{*} \cdot x^{i} \leq p^{*} \cdot e^{\tau i}\right\}$ and for all $x^{i} \in X^{\tau i}$ such that $p^{*} \cdot x^{i}<p^{*} \cdot e^{\tau i}, u^{\tau i}\left(x^{i}\right) \leq u^{\tau i}\left(x^{i *}\right)$,

b) for all $j \in \mathcal{J}$, for all $t=1, \ldots, \tau-1, p^{*} \in \tilde{\varphi}^{\tau j}\left(y^{t j *}\right)$;

c) $\sum_{i \in \mathcal{I}_{0}^{\tau-1}} x^{i *}=\sum_{i \in \mathcal{I}_{0}^{\tau-1}} e^{i}+\sum_{j \in \mathcal{J}} \sum_{t=1}^{\tau-1} y^{t j *}$;

d) $p^{*} \neq 0$.

Proposition 1 Under the assumptions of Theorem 1, for all $\tau \geq 2$, there exists a quasi-equilibrium of the economy $\mathcal{E}_{\tau}$.

Proof. The proof is based on the fact that $\mathcal{E}_{\tau}$ satisfies the necessary assumption of the existence of a (quasi)-equilibrium. See Bonnisseau-Cornet [4] for the existence of equilibrium with bounded-losses pricing rules and in particular of losses-free pricing rules, Gourdel [11] for the existence of quasiequilibrium and the way to go from quasi-equilibrium to equilibrium, and Bonnisseau-Jamin [5] for the existence of equilibrium with a weaker version of the free-disposal assumption.

Indeed, the existence of quasi-equilibrium is ensured by Assumptions (C) and $(\mathrm{E})$, and the facts that :

- $\tilde{\varphi}^{t j}$ satisfies Assumption (PR)(a) since $\varphi_{t}^{j}$ satisfies this assumption and $C_{t}$ is a closed convex cone.

- for all $\left(y^{t j}\right) \in \prod \partial Y^{t j}$, if $p \in \cap_{j, t} \tilde{\varphi}^{t j}\left(y^{t j}\right), p \cdot e^{\tau i}+\sum_{j \in \mathcal{J}} \theta_{t}^{i j} p \cdot y^{t j} \geq 0$, thanks to Assumptions (LF) and (E), and Remark 6, that is $p^{*} \in$ $\prod_{t=1}^{\tau} \mathbb{R}_{+}^{L_{t}}$.

- $Y^{t j}-\prod_{t^{\prime}=1}^{\tau} C_{t^{\prime}}=Y^{t j}$ (free-disposal)

and the boundedness assumption stated by the following lemma.

Let $e \in \prod_{t \in \mathbb{N}^{*}} \mathbb{R}_{+}^{L_{t}}$ defined by $e_{t}=\sum_{i \in \mathcal{I}_{t} \cup \mathcal{I}_{t-1}} e_{t}^{i}$. Let $e^{\prime} \in \prod_{t \in \mathbb{N}^{*}} \mathbb{R}_{+}^{L_{t}}$ such that $e^{\prime} \geq e$. We denote by $\tilde{\mathcal{A}}\left(\mathcal{E}_{\tau}\left(e^{\prime}\right)\right)$, the set of allocations satisfying the market clearing condition for a pseudo-equilibrium (Condition (c) of Definition 2) for the economy $\mathcal{E}_{\tau}$. 
Lemma 1 For all $e^{\prime} \geq e$, for all $j \in \mathcal{J}$, there exists a sequence of non negative real numbers $\left(m^{t j}\right)$ such that for all $\tau$, for all $\left(\left(x^{i}\right),\left(y^{t j}\right)\right) \in \tilde{\mathcal{A}}\left(\mathcal{E}_{\tau}\left(e^{\prime}\right)\right)$,

for all $i \in \mathcal{I}_{0}^{\tau-1}$, for all $t=1, \ldots, \tau-1,0 \leq x_{t}^{i} \leq e_{t}^{\prime}+\left(\sum_{j \in \mathcal{J}} m^{t j}\right) \mathbf{1}^{t}$;

for all $j \in \mathcal{J}$, for all $t=1, \ldots, t-1$, for all $t^{\prime} \neq t+1$,

$0 \geq y_{t^{\prime}}^{t j} \geq-e_{t^{\prime}}^{\prime}-\sum_{j \in \mathcal{J}} m^{t^{\prime} j} \mathbf{1}^{t^{\prime}}$

$m^{(t+1) j} \mathbf{1}^{t+1} \geq y_{t+1}^{t j} \geq-e_{t+1}^{\prime}-\sum_{j \in \mathcal{J}} m^{(t+1) j} \mathbf{1}^{t+1}$.

Proof. Let $\left(\left(x^{i}\right),\left(y^{t j}\right)\right)$ be an element of $\tilde{\mathcal{A}}\left(\mathcal{E}_{\tau}\left(e^{\prime}\right)\right)$. Then, for all $t=1, \ldots, \tau$,

$$
\sum_{i \in \mathcal{I}_{0}^{\tau-1}} x_{t}^{i} \leq e_{t}^{\prime}+\sum_{j \in \mathcal{J}} \sum_{t^{\prime}=1}^{\tau-1} y_{t}^{t^{\prime} j}
$$

For all $j \in \mathcal{J}$, we define the sequence $\left(m^{t j}\right)$ as follows: $m^{1 j}=0$ and $m^{t+1 j}$ is a positive real number so that:

$$
F_{t}^{j}\left(-e_{t}^{\prime}-\sum_{j \in \mathcal{J}} m^{t j} \mathbf{1}^{t}\right) \subset m^{t+1 j} \mathbf{1}^{t+1}-\mathbb{R}_{+}^{L_{t+1}}
$$

Such real number exists from the boundedness Assumption F(c). Since $0 \leq$ $\sum_{i \in \mathcal{I}_{0}^{\tau-1}} x_{1}^{i}$, we get $\sum_{j \in \mathcal{J}} y_{1}^{1 j}+\sum_{j \in \mathcal{J}} \sum_{t=2}^{\tau-1} y_{1}^{t j} \geq-e_{1}^{\prime}$. Since for all $j \in \mathcal{J}$, for all $t=1, \ldots, \tau-1, y_{1}^{t j} \leq 0$, we obtain $0 \geq y_{1}^{1 j} \geq-e_{1}^{\prime}$ for all $j$ and for all $t$.

For the second period, we have

$$
\sum_{j \in \mathcal{J}} y_{2}^{1 j}+\sum_{j \in \mathcal{J}} y_{2}^{2 j}+\sum_{j \in \mathcal{J}} \sum_{t=3}^{\tau-1} y_{2}^{t j} \geq-e_{2}^{\prime}
$$

For all $j \in \mathcal{J}$, for all $t=2, \ldots, \tau-1, y_{2}^{t j} \leq 0$. From the above inequalities and Assumption $\mathrm{F}(\mathrm{d}), y_{2}^{1 j} \in F_{1}^{j}\left(y_{1}^{1 j}\right) \subset F_{1}^{j}\left(-e_{1}^{\prime}\right) \subset m^{2 j} \mathbf{1}^{2}-\mathbb{R}_{+}^{L_{2}}$. Thus, for all $j \in \mathcal{J}$,

$$
\begin{gathered}
0 \geq y_{2}^{t j} \geq-e_{2}^{\prime}-\sum_{j \in \mathcal{J}} m^{2 j} \mathbf{1}^{2}, \text { for all } t=2, \cdots, \tau-1, \\
m^{2 j} \mathbf{1}^{2} \geq y_{2}^{1 j} \geq-e_{2}^{\prime}-\sum_{j \neq j^{\prime}} y_{2}^{1 j^{\prime}} \geq-e_{2}^{\prime}-\sum_{j \neq j^{\prime}} m^{2 j^{\prime}} \mathbf{1}^{2} \geq-e_{2}^{\prime}-\sum_{j^{\prime} \in \mathcal{J}} m^{2 j^{\prime}} \mathbf{1}^{2}
\end{gathered}
$$

By an induction argument taking into account the definition of the sequences $\left(\mathrm{m}^{t j}\right)$ we prove the result for all periodS. 
For the consumptions, since they are all non-negative,

$$
0 \leq x^{i} \leq \sum_{i^{\prime} \in \mathcal{I}_{0}^{\tau-1}} x^{i^{\prime}} .
$$

So, for all $t$

$$
0 \leq x_{t}^{i} \leq e_{t}^{\prime}+\sum_{j \in \mathcal{J}} \sum_{t^{\prime}=1}^{\tau-1} y_{t}^{t^{\prime} j} \leq e_{t}^{\prime}+\sum_{j \in \mathcal{J}} y_{t}^{t-1 j} \leq e_{t}^{\prime}+\sum_{j \in \mathcal{J}} m^{t j} \mathbf{1}^{t}
$$

The following lemma ensures that a quasi-equilibrium in $\mathcal{E}_{\tau}$ is an equilibrium.

Lemma 2 If $\left(p^{*},\left(x^{i *}\right),\left(y^{t j *}\right)\right)$ is a quasi-equilibrium, then $p_{t}^{*} \neq 0$ for all $t$ and $\left(p^{*},\left(x^{i *}\right),\left(y^{t j *}\right)\right)$ is an equilibrium.

Proof. Since the utility functions are continuous, the condition for a quasiequilibrium $\left(p^{*},\left(x^{i *}\right),\left(y^{t j *}\right)\right)$ to be an equilibrium is that the indidvidual wealth is strictly above the subsistence level, that is: $w^{i *}=p^{*} \cdot e^{\tau i}+$ $\sum_{j \in \mathcal{J}} \theta_{t}^{i j} p^{*} \cdot y^{t j *}>\inf p^{*} . X^{\tau i}$, for all $i \in \mathcal{I}_{0}^{\tau-1}$. As already remarked (See Remark 6), $p^{*} \in \prod_{t=1}^{\tau} \mathbb{R}_{+}^{L_{t}}$, so inf $p^{*} . X^{\tau i}=0$. Hence, from Assumptions E and LF, it suffices to show that $p_{t}^{*} \neq 0$ for all $t=1, \ldots, \tau$.

Suppose that there exists $t$ such that $p_{t}^{*}=0$. Knowing that $p^{*}$ is not equal to 0 , there exists $\bar{t}$ such that $p_{\bar{t}}^{*} \neq 0$ and $p_{\bar{t}+1}^{*}=0$ or $p_{\bar{t}}^{*}=0$ and $p_{\bar{t}+1}^{*} \neq 0$. We deal with the first case, the proof being the same for the second case.

Since $p_{\bar{t}}^{*} \in \mathbb{R}_{+}^{L_{\bar{t}}} \backslash\{0\}$, the consumer $i_{1}$ in $\mathcal{I}_{\bar{t}}$ given by Assumption $\mathrm{C}(\mathrm{c})$ has a strictly positive wealth $w^{i_{1} *}>0$. Then $\left(x_{\bar{t}}^{i_{1} *}, x_{\bar{t}+1}^{i_{1} *}\right)$ is a demand of consumer $i_{1}$. But, then, the local non-satiation of the partial utility function $u^{i_{1}}\left(x_{\bar{t}}^{i_{1} *}, \cdot\right)$ is incompatible with $p_{\bar{t}+1}^{*}=0$.

Thus, necessarily $p_{t}^{*} \neq 0$ for all $t$, and $w^{i *}>\inf p^{*} \cdot X^{\tau i}=0$.

From Remark 2, an equilibrium is a pseudo-equilibrium, thus we have proved the following result.

Proposition 2 Under the assumptions of Theorem 1, for all $\tau \geq 2$, there exists a pseudo-equilibrium of the economy $\mathcal{E}_{\tau}$.

In the following lemma, we provide two properties of the pseudo-equilibrium, which will be useful for the limit argument in the next section. A non zero equilibrium price $p^{*}$ is normalized so that $\sum_{t=1}^{\tau} \sum_{\ell \in L_{t}} p_{t \ell}^{*}=1$.

Lemma 3 a) If $\left(p^{*},\left(x^{i *}\right),\left(y^{t j *}\right)\right)$ is a pseudo-equilibrium, then $p_{t}^{*} \neq 0$ for all $t$. 
b) The set of pseudo-equilibria of the economy $\mathcal{E}_{\tau}$ with a normalized price is closed.

Proof. The first part uses the same argument as for Lemma 2.

We now consider a sequence of pseudo-equilibria $\left(p^{\nu},\left(x^{i \nu}\right),\left(y^{t j \nu}\right)\right)$ that converges to $\left(\bar{p},\left(\bar{x}^{i}\right),\left(\bar{y}^{t j}\right)\right)$. We prove that $\left(\bar{p},\left(\bar{x}^{i}\right),\left(\bar{y}^{t j}\right)\right)$ is also a pseudoequilibrium.

It is easy to establish that $\left(\bar{p},\left(\bar{x}^{i}\right),\left(\bar{y}^{t j}\right)\right.$ satisfies Condition (b) in Definition 2, since $\tilde{\varphi}^{\tau j}$ has closed graph, and also Condition (c). So it remains to show that the condition (a) is also satisfied.

Let us denote by $\left(w^{i \nu}\right)$ the associated wealth sequence and by $\bar{w}^{i}$ its limit. One easily shows that the budget constraint is satisfied by $\bar{x}^{i}$. If $\bar{w}^{i}>0$, then $\bar{x}^{i}$ maximizes the utility function under the budget constraint. Indeed, if $\bar{p} \cdot x^{i}<\bar{w}^{i}$, then for $\nu$ large enough, $p^{\nu} \cdot x^{i} \leq w^{i \nu}$. But this implies that $u^{i}\left(x^{i}\right) \leq u^{i}\left(x^{i \nu}\right)$, and by the continuity of $u^{i}, u^{i}\left(x^{i}\right) \leq u^{i}\left(\bar{x}^{i}\right)$. If $\bar{p} \cdot x^{i}=\bar{w}^{i}>0$. Let $\lambda<1$. Then $\bar{p} \cdot\left(\lambda x^{i}\right)<\bar{w}^{i}$. So, from above, $u^{i}\left(\lambda x^{i}\right) \leq u^{i}\left(\bar{x}^{i}\right)$. Using again the continuity of $u^{i}, u^{i}\left(x^{i}\right)=\lim _{\lambda \rightarrow 1} u^{i}\left(\lambda x^{i}\right) \leq u^{i}\left(\bar{x}^{i}\right)$.

Let us now prove that $\bar{p}_{t} \neq 0$, for all $t$. Since $\bar{p} \neq 0$ by normalization, there exists $t$ such that $\bar{p}_{t} \neq 0$. Hence, for the consumer $i_{0}(t) \in \mathcal{I}_{t}$ and $i_{1}(t-1) \in \mathcal{I}_{t-1}, \bar{w}^{i_{0}(t)}>0$ and $\bar{w}^{i_{1}(t-1)}>0$. So the agents $i_{0}(t)$ and $i_{1}(t-1)$ are utility maximizer hence, from Assumption $\mathrm{C}(\mathrm{c}), \bar{p}_{t+1} \neq 0$ and $\bar{p}_{t-1} \neq 0$. Doing recursively the same argument, we conclude that the prices at each period is different from 0 .

Since $\bar{p}_{t} \neq 0$, for all $t, \bar{w}^{i}>0$ for all consumers, hence all of them are maximizing utility at the price $\bar{p}$.

\section{From truncated equilibria to equilibrium}

The proof of Theorem 1 consists of considering a sequence of pseudo-equilibrium in the truncated economy with an horizon increasing to infinity. First, we establish that the sequence of equilibrium prices in the truncated economies remains in a compact set for the product topology on $\prod_{t=1}^{\infty} \mathbb{R}^{L_{t}}$. Then we show that the sequence of $T$-equilibrium remains in a compact set and we prove that a cluster point is an equilibrium of the OLG economy $\mathcal{E}$.

From the previous section, for all $T \geq 2$, there exists a $T$-equilibrium $\left(p^{T},\left(x^{i T}\right),\left(y^{t j T}\right)\right)$ of the economy $\mathcal{E}_{T}$. Since we have proved in the previous section (see Lemma 2) that $p_{1}^{T} \neq 0$, we normalize $p^{T}$ so that $\sum_{\ell \in L_{1}} p_{1 \ell}^{T}=1$.

We extend the price and the allocations to the whole space $\prod_{t=1}^{\infty} \mathbb{R}^{L_{t}}$ by 
adding zeros for the missing components without modifying the notations. So, now the sequences $\left(p^{T}\right),\left(x^{i T}\right)$ and $\left(y^{t j T}\right)$ are in $\prod_{t=1}^{\infty} \mathbb{R}^{L_{t}}$.

We now prove that the sequence of prices $\left(p^{T}\right)$ remains in a compact subset of $\prod_{t=1}^{\infty} \mathbb{R}^{L_{t}}$.

Lemma 4 For all $t$, there exists $\tilde{k}_{t} \in \mathbb{R}_{+}$such that for all $T, 0 \leq p_{t}^{T} \leq \tilde{k}_{t} \mathbf{1}^{t}$.

Proof. If it is not true, there exist $\bar{t}$ and an increasing sequence $\left(T^{\nu}\right)$ such that $p_{\bar{t}}^{T^{\nu}} \geq \nu \mathbf{1}^{\bar{t}}$. Let $\tau>\bar{t}+2$. We assume without any loss of generality that $T^{\nu}>\tau$ for all $\nu$.

Now we consider the restriction to the $\tau$ first period of the $T^{\nu}$-equilibrium $\left(p^{T^{\nu}},\left(x^{i T^{\nu}}\right),\left(y^{t j T^{\nu}}\right)\right)$ :

- for all $i \in \mathcal{I}_{0}^{\tau-1}, x^{i \nu}$ is the restriction of $x^{i T^{\nu}}$ to $\prod_{t=1}^{\tau} \mathbb{R}^{L_{t}}$;

- for all $j \in \mathcal{J}$, for all $t=1, \ldots, \tau-1, y^{t j \nu}$ is the restriction of $y^{t j T^{\nu}}$ to $\prod_{t=1}^{\tau} \mathbb{R}^{L_{t}}$

- $p^{\nu}$ is the restriction of $p^{T^{\nu}}$ to $\prod_{t=1}^{\tau} \mathbb{R}^{L_{t}}$.

From Remark 7 in the previous section, $\left(p^{\nu},\left(x^{i \nu}\right),\left(y^{t j \nu}\right)\right)$ is a pseudoequilibrium of the truncated economy $\mathcal{E}_{\tau}$. We now renormalize the price $p^{\nu}$ as follows:

$$
\pi^{\nu}=\frac{1}{\sum_{t=1}^{\tau} \sum_{\ell \in L_{t}} p_{t \ell}^{\nu}} p^{\nu}
$$

Since $\pi^{\nu}$ is non negative, the sequence $\pi^{\nu}$ remains in the simplex of $\prod_{t=1}^{\tau} \mathbb{R}^{L_{t}}$, which is compact. From Lemma 1 in the previous section, the sequence $\left(\left(x^{i \nu}\right),\left(y^{t j \nu}\right)\right)$ remains in the compact subset $\tilde{\mathcal{A}}\left(\mathcal{E}_{\tau}(e)\right)$. So the sequence $\left(\pi^{\nu},\left(x^{i \nu}\right),\left(y^{t j \nu}\right)\right)$ has a cluster point $\left(\bar{\pi},\left(\bar{x}^{i}\right),\left(\bar{y}^{t j}\right)\right)$. From Lemma 3 , this cluster point $\left(\bar{\pi},\left(\bar{x}^{i}\right),\left(\bar{y}^{t j}\right)\right)$ is also a pseudo-equilibrium of the truncated economy $\mathcal{E}_{\tau}$. But $\bar{\pi}_{1}=0$ since $\left(\sum_{t=1}^{\tau} \sum_{\ell \in L_{t}} p_{t \ell}^{\nu}\right)$ converges to $+\infty$ and $0 \leq p_{1 \ell}^{\nu} \leq 1$ for all $\ell \in L_{1}$. Hence we get a contradiction since Lemma 3 shows that for all $t=1, \ldots, \tau, \bar{\pi}_{t} \neq 0$.

Proof of Theorem 1. From Lemma 1 and the above lemma, the sequence of $T$-equilibrium of the economy $\mathcal{E}_{T},\left(p^{T},\left(x^{i T}\right),\left(y^{t j T}\right)\right)$, remains in a compact set for the product topology of $\prod_{t=1}^{\infty} \mathbb{R}^{L_{t}} \times \prod_{t^{\prime}=1}^{\infty} \prod_{i \in \mathcal{I}_{t^{\prime}}} \prod_{t=1}^{\infty} \mathbb{R}^{L_{t}} \times$ $\prod_{j \in \mathcal{J}} \prod_{t^{\prime}=1}^{\infty} \prod_{t=1}^{\infty} \mathbb{R}^{L_{t}}$. Since this is a countable product of finite dimensional spaces, the product topology is metrizable on the compact sets and there exists a sub-sequence $\left(p^{T^{\nu}},\left(x^{i T^{\nu}}\right),\left(y^{t j T^{\nu}}\right)\right)$ of $\left(p^{T},\left(x^{i T}\right),\left(y^{t j T}\right)\right)$, which converges 
to $\left(p^{*},\left(x^{i *}\right),\left(y^{t j *}\right)\right)$. We recall that the convergence for the product topology implies the usual convergence when we consider only a finite number of components.

For each $\tau \geq 2$, for $\nu$ large enough, the restriction of $\left(p^{T^{\nu}},\left(x^{i T^{\nu}}\right),\left(y^{t j T^{\nu}}\right)\right)$ to the $\tau$ first periods is a pseudo-equilibrium of $\mathcal{E}_{\tau}$ (see Remark 7 ) and it converges to the restriction of $\left(p^{*},\left(x^{i *}\right),\left(y^{t j *}\right)\right)$ to the $\tau$ first periods. From Lemma 3, this restriction is a pseudo-equilibrium of $\mathcal{E}_{\tau}$. From Definition 2 and the notations above, one deduces that $\left(p^{*},\left(\xi^{i *}\right),\left(y^{j *}\right)\right)$ defined as follows in an equilibrium for the OLG economy $\mathcal{E}$ :

- for all $t \geq 1$, for all $i \in \mathcal{I}_{t}, \xi^{i *}=\left(x_{t}^{i *}, x_{t+1}^{i *}\right)$ and for all $i \in \mathcal{I}_{0}, \xi^{i *}=x_{1}^{i *}$;

- for all $j \in \mathcal{J}$ for all $t \geq 1, z_{t}^{j *}=y_{t}^{t j *}, \zeta_{t+1}^{j *}=y_{t+1}^{t j *}$ and $y_{t}^{j *}=z_{t}^{j *}+\zeta_{t}^{j *}$ with $\zeta_{0}^{j *}=0$.

\section{References}

[1] Balasko, Y., Cass D. and Shell K., Existence of Competitive Equilibrium in a General Overlapping Generations Model, Journal of Economic Theory, 23, $307-322,1980$.

[2] Balasko Y. and Shell K., The Overlapping Generations Model I: The Case of Pure Exchange without Money, Journal of Economic Theory, $23,281-306,1980$.

[3] Balasko Y. and K. Shell K., The Overlapping Generations Model II: The Case of Pure Exchange with Money, Journal of Economic Theory, 24, 112 - 142, 1981.

[4] Bonnisseau, J.M. and Cornet B., Existence of Equilibria when Firms Follow Bounded Losses Pricing Rules, Journal of Mathematical Economics, 17, $119-147,1988$.

[5] Bonnisseau, J.M. and Jamin A., Equilibria with Increasing Returns: Sufficient Conditions on Bounded Production Allocations, Journal of Public Economic Theory, 10, 6, 1033 - 1068, 2008.

[6] Bonnisseau, J.M. and Jamin A., The Survival Assumption in Intertemporal Economies, The B.E. Journal of Theoretical Economics: Vol. 11 : Iss. 1 (Contributions), Article 14. DOI: 10.2202/1935-1704.1781, 2011.

[7] Cornet, B., General equilibrium theory and increasing returns: presentation, Journal of Mathematical Economics, 17, 103 - 118, 1988. 
[8] Dehez, P. and Drèze, J., Competitive equilibria with quantity-taking producers and increasing returns to scale, Journal of Mathematical Economics , 17, 209 - 230, 1988.

[9] Dehez, P. and Drèze, J., Distributive production sets and equilibria with increasing returns, Journal of Mathematical Economics, 17, 231 - 248, 1988 .

[10] Dierker, E., Guesnerie, R. and Neuefeind, W., General equilibrium where some firms follow special pricing rules, Econometrica, 53, 1369 - 1393, 1985 .

[11] Gourdel, P., Existence of Intransitive Equilibria in Nonconvex Economies, Set-Valued Analysis, 3, 307 - 337, 1995.

[12] Quinzii M., Rendements croissants et efficacité économique, Monographie d'économétrie, Editions du CNRS, Paris, 1988.

[13] Samuelson, P.A., An Exact Consumption-Loan Model of Interest with or without the Social Contrivance of Money, Journal of Political Economy, 66, 467-482, 1958.

[14] Tvede, M., Overlapping Generations Economies, Palgrave Macmillan, 2010 .

[15] Villar, A., Equilibrium and Efficiency in Production Economies, Second Revised and Enlarged Edition, Springer-Verlag Berlin, Heidelberg, 2000. 